\title{
Dipteryx alata Seedlings Nutritional Status in a Recovery Area in the Brazilian Savannah
}

\author{
Thaís Soto Boni ${ }^{1}$ (D) 0000-0001-7201-6122 \\ Kátia Luciene Maltoni ${ }^{1}$ [D 0000-0001-6619-4504 \\ Kellian Kenji Gonzaga da Silva Mizobata ${ }^{1}$ (1) 0000-0001-6350-4466
}

\begin{abstract}
Dipteryx alata is an arboreal species found in the Brazilian Savannah (BS), that has potential human and animal uses, including nutrition, medicine, and revegetation. This study aimed to evaluate the nutritional status and growth of D. alata seedlings used to recover degraded soils to which residues had been added compared to seedlings grown in the BS. The degraded soil was treated with organic and agroindustrial residues. Twelve months after seedlings were introduced, they were evaluated for height, stem diameter, chlorophyll index, and concentration of foliar macro and micronutrients. The foliar concentration was determined for D. alata seedlings grown in the degraded area and BS. The addition of residues increased foliar concentration of seedlings grown in the degraded area; however, concentrations did not reach similar levels to seedlings grown in BS. D. alata accumulated Mn in plants. The height and stem diameter of seedlings responded positively to residue application.
\end{abstract}

Keywords: degraded soil, residue, macrophytes, ash from sugarcane bagasse.

\section{INTRODUCTION AND OBJECTIVES}

The combination of high species richness and high degree of threat has resulted in the Brazilian Savannah (Neotropical savannah), known as Cerrado, being included in the list of biodiversity hotspots considered critical for biodiversity conservation worldwide (Myers et al., 2000; Sloan et al., 2014).

In the Brazilian Savannah, South of Midwest region and Eastern of Mato Grosso do Sul, in 1965 a hydroelectric power plant was built, generating extensive degraded areas (borrow areas), from which vegetation and first soil horizons were removed, reaching $10 \mathrm{~m}$ deep in some regions. Even after 50 years, few natural vegetation has regrown in these areas. In degradation areas where clearcutting and earthworks occurred, the vegetation of the Brazilian Savannah has presented only low to intermediate revegetation potential (Carnevale \& Montagnini, 2002).

Besides being devoid of physical attributes that could facilitate spontaneous revegetation, the substrate of these areas is chemically poor and susceptible to erosive processes (Pedrol et al., 2010; Rodrigues et al., 2007; Zheng, 2006). Under such poor nutrient conditions, revegetation with native species has been recommended. These species tend to be better adapted to environmental conditions and, thus, have greater chance of survival (Tang \& Li, 2014). In this sense, the use of chemical and organic inputs are necessary to improve the chemical, physical, and biological conditions of degraded soil, and to facilitate the reintroduction of vegetation. Such inputs are added to focal areas that have a pronounced dry season and low fertility (Pedrol et al., 2010). A variety of organic and agricultural residues are used under such conditions. Examples include aquatic macrophytes and the ash of sugarcane bagasse. The residues of macrophytes contain important elements, such as $\mathrm{Ca}, \mathrm{Mg}, \mathrm{K}, \mathrm{N}, \mathrm{P}$, and $\mathrm{C}$ in the form of organic matter in macrophytes (Gunnarsson \& Petersen, 2007) and as calcinated material in ash (Novak et al., 2009).

The selection of a single or multiple plant species for revegetation is important, and it must comprehend characteristics of the species and the area where they will be introduced. Dipteryx alata Vogel, popularly known as Baru, is a common leguminous tree in the Brazilian Savannah (Lorenzi, 2000; Sano et al., 2004), and it is one of the few species that have fruits with a fleshy pulp during the dry season, providing an

\footnotetext{
${ }^{1}$ Universidade Estadual Paulista "Júlio de Mesquita Filho" (Unesp), Ilha Solteira, SP, Brasil
} 
important source of food for wildlife during this period. D. alata is commercially interesting because its nuts, which are rich in proteins, lipids, calcium, iron, and zinc, as well as phenolic acids, phytic acid, and tannin, all of which are important for human health (Lemos et al., 2012; Siqueira et al., 2012). As a zoochorous plant, it attracts fruit-eating animals and could contribute to the recovery of degraded areas and enrich the local diversity by promoting the dispersal of its seeds, as well as the seeds from other plants (Reid et al., 2015).

The production of seedlings is an important phase in revegetation projects. Good quality seedlings and suitable substrates are essential to ensure successful adaptation and growth after seedlings are introduced to sites (Le et al., 2014). Knowledge about the nutritional requirements of $D$. alata remains limited. According to Silva et al. (2015), the addition of poultry litter and green manure increases the mortality of the seedlings of plants from the Brazilian Savannah, including $D$. alata. Specifically, the seedlings of $D$. alata were more likely to establish without fertilization; thus, establishment of this species in field is not only dependent on soil fertility. Actually, the effect of fertilization on the establishment of $D$. alata seedlings might differ on severely degraded soils.

Therefore, this study compared the nutritional status and growth of $D$. alata seedlings introduced to degraded area treated with residue (organic and agroindustrial) versus a conserved area of Brazilian Savannah.

\section{MATERIALS AND METHODS}

In November 2011 at the Unesp/Ilha Solteira Campus Experimental Farm, located in Selvíria, MS, Brazil, a recovery project was established on an experimental area of $34,000 \mathrm{~m}^{2}$ $\left(20^{\circ} 23^{\prime} 2^{\prime \prime} \mathrm{S}\right.$ and $\left.51^{\circ} 24^{\prime} 24^{\prime \prime} \mathrm{W}\right)$ where up to $10 \mathrm{~m}$ depth soil had been removed, 50 years ago. The average altitude of the area is $335 \mathrm{~m}$. This region weather was classified as Aw (tropical savanna climate with dry winter) according to the Köppen's climate classification, with temperature and precipitation annual means of $23.7^{\circ} \mathrm{C}$ and $1,300 \mathrm{~mm}$, respectively (Figure 1). The predominant soil in this region is Typic Hapludox.

The experimental design was a randomized block with agroindustrial residues (AR) being applied in bands, in a factorial $3 \times 4$. Three quantities $\left(0,16\right.$, and $\left.32 \mathrm{Mg} \mathrm{ha}^{-1}\right)$ of organic residue (OR) from macrophytes were applied and four quantities $\left(0,15,30\right.$, and $\left.45 \mathrm{Mg} \mathrm{ha}^{-1}\right)$ of agroindustrial residue (AR) ash from sugarcane bagasse were applied. In total, 12 treatments with 3 replicates occured, and they were established in 36 blocks of $600 \mathrm{~m}^{2}$ each.

The OR was collected from the same region, and it was composed of a mixture of Egeria densa Planch., Egeria najas Planch., Ceratophyllum demersum L., Eichhornia azurea Kunth, Eichhornia crassipes (Mart.) Solms., Pistia stratiotes L., and Typha latifolia L., as reported by Thomaz et al. (2008) for the hydroelectric power plant Jupiá in Três Lagoas, MS. The AR was collected from a boiler plant Alcoolvale: Açúcar e Álcool S.A. in Aparecida do Taboado, MS. The residues were exposed to the sun for 120 days, and then they were incorporated into the degraded soil.

On February 2012, the seedlings of 10 species found in the remaining Brazilian Savannah near the experimental area were introduced to the experimental area. The plants were placed in $0.40 \mathrm{~m}$ depth pits of $4.0 \times 5.0 \mathrm{~m}$ spacing. A total of 1,080 seedlings were introduced. Each block received three individuals of each species. D. alata was one of these species, with seedlings of 0.20 and $0.30 \mathrm{~m}$ high being used.

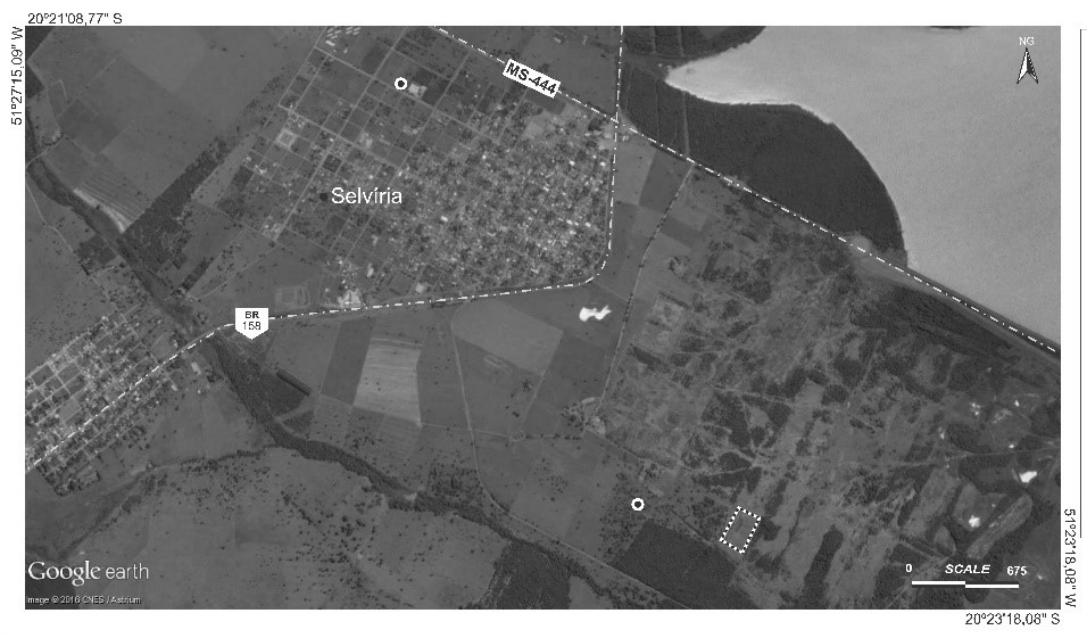

Mato Grosso do Sul - MS

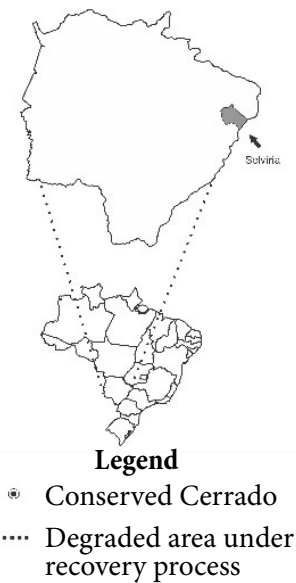

Figure 1. Location of the experimental area (degraded area under recovery process) and conserved Cerrado area where leaf samples were collected. 
The seedlings grown from seeds collected in the nearby Brazilian Savannah and were subjected to a pre-germination treatment. The pericarp (exocarp, mesocarp, and endocarp) was mechanically removed with a hammer and pliers. The seeds were germinated in bags containing substrate composed of soil from the degraded area mixed with AR in a 1:3 ratio (soil:ash). After 8 months, the seedlings were transferred to the field. After 30 days, the seedlings that did not survive were replaced. Seedlings were irrigated weekly during March, July, August, and October, which encompassed the driest period of the first year (Figure 2), to prevent death.

In February 2013, one year later, the nutritional status of D. alata was assessed by determining the foliar concentrations of N, P, K, Ca, Mg, S, B, Cu, Fe, Mn, and Zn. Three leaves from each plant were combined to form one sample for each block. These plants were also assessed for height $(\mathrm{cm})$ and stem diameter $(\mathrm{mm})$. The soil chemicals were characterized (Agbenin et al., 1999; Raij et al., 1986) in composite samples (five single samples) collected at 0.0 to $0.20 \mathrm{~m}$ depth in each block, with three replicates per treatment, producing a total of 36 samples.

In total, five seedlings of $D$. alata (each sample contained three sub-samples) were also collected from fragments of conserved Brazilian Savannah (CER) to compare the nutritional status of seedlings inserted in the experimental area. The seedlings were collected during February 2014, dried and subjected to the same foliar analysis. In addition to collecting seedlings from CER, three soil replicates ( 0 to $20 \mathrm{~cm}$ depth) were collected from which P, OM, pH, K, Ca, $\mathrm{Mg}, \mathrm{H}+\mathrm{Al}, \mathrm{Al}, \mathrm{Cu}, \mathrm{Fe}, \mathrm{Mn}$, and $\mathrm{Zn}$ values were determined by ion exchange resins (Agbenin et al., 1999; Raij et al., 1986). Table 1 shows the characteristics of the residues.

The data were evaluated by analysis of variance, followed by Dunnett's test $(p<0.05)$ and regression analysis using SAS 9.4 statistical software (SAS, 2012).

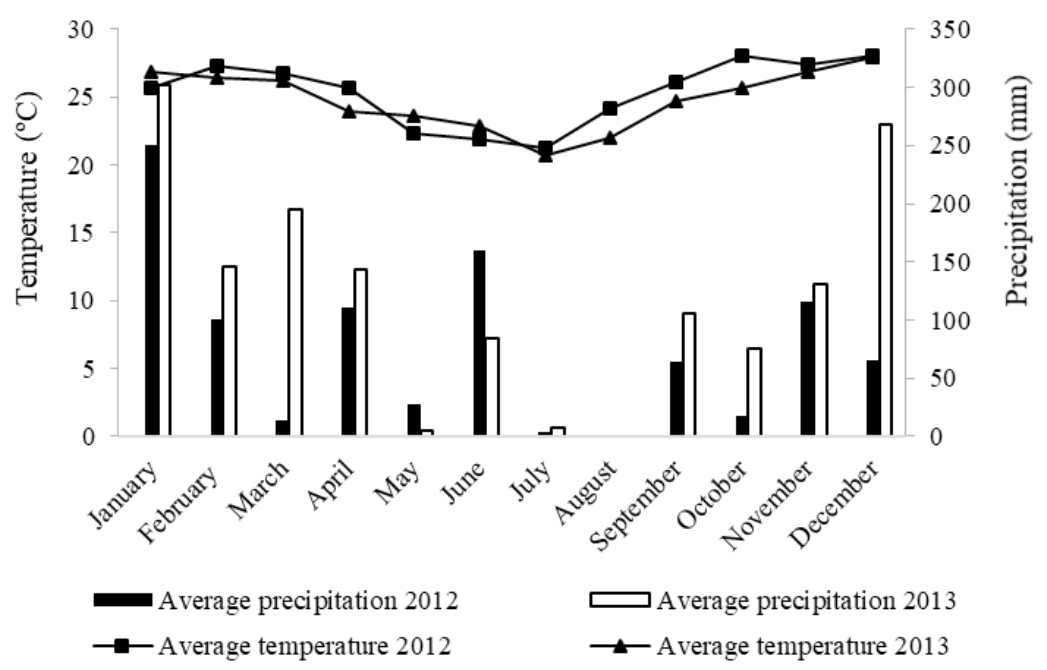

Figure 2. Monthly average temperature and precipitation in 2012 and 2013.

Source: Canal Clima da Unesp Ilha Solteira ([2015?]).

Table 1. Chemical characterization of organic residue and the physical chemical characterization of agroindustrial residue.

\begin{tabular}{cccccc} 
& Organic residue & & \multicolumn{3}{c}{ Agroindustrial residue } \\
\hline Parameter & Unit & Result & Parameter & Unit & Result \\
\hline Nitrogen & $\mathrm{g} \mathrm{kg}^{-1}$ & 17.6 & Barium & $\mathrm{mg} \mathrm{kg}^{-1}$ & 25.7 \\
Phosphorus & $\mathrm{g} \mathrm{kg}^{-1}$ & 1.7 & Sodium & $\mathrm{mg} \mathrm{kg}^{-1}$ & 18.0 \\
Potassium & $\mathrm{g} \mathrm{kg}^{-1}$ & 6.5 & Potassium & $\mathrm{mg} \mathrm{kg}^{-1}$ & 1617 \\
Calcium & $\mathrm{g} \mathrm{kg}^{-1}$ & 11.6 & Arsenio & $\mathrm{mg} \mathrm{kg}^{-1}$ & $<1.0$ \\
Magnesium & $\mathrm{g} \mathrm{kg}^{-1}$ & 2.4 & Selenium & $\mathrm{mg} \mathrm{kg}^{-1}$ & $<1.0$ \\
Sulfur & $\mathrm{g} \mathrm{kg}^{-1}$ & 6.7 & Mercury & $\mathrm{mg} \mathrm{kg}^{-1}$ & $<1.0$ \\
Boron & $\mathrm{mg} \mathrm{kg}^{-1}$ & 27 & Aluminum & $\mathrm{mg} \mathrm{kg}^{-1}$ & 1710 \\
Copper & $\mathrm{mg} \mathrm{kg}^{-1}$ & 57 & Boron & $\mathrm{mg} \mathrm{kg}^{-1}$ & $<3.2$ \\
Iron & $\mathrm{mg} \mathrm{kg}^{-1}$ & 2000 & Cadmium & $\mathrm{mg} \mathrm{kg}^{-1}$ & $<0.4$ \\
\hline
\end{tabular}


Table 1. Continued...

\begin{tabular}{|c|c|c|c|c|c|}
\hline \multicolumn{3}{|c|}{ Organic residue } & \multicolumn{3}{|c|}{ Agroindustrial residue } \\
\hline Parameter & Unit & Result & Parameter & Unit & Result \\
\hline Manganese & $\mathrm{mg} \mathrm{kg}^{-1}$ & 194 & Calcium & $\mathrm{g} \mathrm{kg}^{-1}$ & 5.3 \\
\hline Zinc & $\mathrm{mg} \mathrm{kg}^{-1}$ & 34 & Lead & $\mathrm{mg} \mathrm{kg}^{-1}$ & 3.4 \\
\hline \multicolumn{3}{|c|}{ Agroindustrial residue } & Copper & $\mathrm{mg} \mathrm{kg}{ }^{-1}$ & 21.1 \\
\hline Parameter & Unit & Result & Chrome & $\mathrm{mg} \mathrm{kg}^{-1}$ & 12.5 \\
\hline $\mathrm{pH}\left(\mathrm{H}_{2} 01: 10\right)$ & - & 5.2 & Sulfur & $\mathrm{g} \mathrm{kg}^{-1}$ & 0.24 \\
\hline Humidity $\left(60-65^{\circ} \mathrm{C}\right)$ & $\%(\mathrm{~m} / \mathrm{m})$ & 3.2 & Iron & $\mathrm{mg} \mathrm{kg}^{-1}$ & 1540 \\
\hline Total solids & $\%(\mathrm{~m} / \mathrm{m})$ & 95.2 & Phosphor & $\mathrm{g} \mathrm{kg}^{-1}$ & 0.86 \\
\hline Volatile solids & $\%(\mathrm{~m} / \mathrm{m})$ & 48.7 & Magnesium & $\mathrm{g} \mathrm{kg}^{-1}$ & 1.1 \\
\hline C Organic & $\mathrm{g} \mathrm{kg}^{-1}$ & 570 & Manganese & $\mathrm{mg} \mathrm{kg}^{-1}$ & 182 \\
\hline N Kejldahl & $\mathrm{g} \mathrm{kg}^{-1}$ & 6.1 & Molybdenum & $\mathrm{mg} \mathrm{kg}^{-1}$ & $<0.9$ \\
\hline N Ammoniacal & $\mathrm{mg} \mathrm{kg}^{-1}$ & 220 & Nickel & $\mathrm{mg} \mathrm{kg}^{-1}$ & $<2.4$ \\
\hline N Nitrate-Nitrite & $\mathrm{mg} \mathrm{kg}^{-1}$ & 421 & Zinc & $\mathrm{mg} \mathrm{kg}^{-1}$ & 12.4 \\
\hline
\end{tabular}

\section{RESULTS}

The comparative analysis of the nutritional status of $D$. alata from the experimental area versus the conserved CER presented no significant differences in the foliar concentrations of $\mathrm{Ca}, \mathrm{Mg}$, and $\mathrm{Cu}$ (Table 2), and considering only the experimental area, $\mathrm{N}, \mathrm{P}, \mathrm{Cu}$, and $\mathrm{Mn}$ foliar concentration present no significant differences (Table 3).

$\mathrm{Fe}, \mathrm{Mn}$ and $\mathrm{Zn}$ concentrations were lower in leaves of plants from CER compared to the experimental area, where seedlings showed higher concentrations ranging from $270 \%$ to $565 \%$ for $\mathrm{Mn}, 125 \%$ to $744 \%$ for $\mathrm{Fe}$, and $155 \%$ to $656 \%$ for $\mathrm{Zn}$ (Table 2).
There was broad variation among treatments, preventing the detection of any trends for these parameters (Tables 2 and 3).

Considering macronutrients, $\mathrm{N}, \mathrm{P}$, and $\mathrm{K}$ concentrations were higher in seedlings collected in CER, in comparison to the experimental area. In this area the $\mathrm{N}$ concentration was higher where $16 \mathrm{Mg} \mathrm{ha}^{-1} \mathrm{OR}$ was introduced. However, the $\mathrm{N}$ concentration was lower compared to the seedlings from the CER $\left(25 \mathrm{~g} \mathrm{~kg}^{-1}\right)$. Notably, the $16 \mathrm{Mg} \mathrm{ha}^{-1}$ dose, rather than the largest dose $\left(32 \mathrm{Mg} \mathrm{ha}^{-1}\right)$, enhanced $\mathrm{N}$ concentrations in D. alata, suggesting that this is not a very $\mathrm{N}$ demanding plant (Table 2 and 3).

Table 2. Mean values of foliar concentration of $\mathrm{N}, \mathrm{P}, \mathrm{K}, \mathrm{Ca}, \mathrm{Mg}, \mathrm{S}, \mathrm{B}, \mathrm{Cu}, \mathrm{Fe}, \mathrm{Mn}$, and $\mathrm{Zn}$ in Dipteryx alata seedlings in relation to the residue treatments at the experimental site versus the natural sites (remnants of Cerrado). F values and coefficient of variation $(\mathrm{CV})$ are also presented in the Table.

\begin{tabular}{|c|c|c|c|c|c|c|c|c|c|c|}
\hline \multirow{2}{*}{ Treatments } & $\mathbf{N}$ & $\mathbf{P}$ & $\mathbf{K}$ & $\mathrm{Ca}$ & $\mathrm{Mg}$ & B & $\mathbf{C u}$ & $\mathrm{Fe}$ & Mn & $\mathrm{Zn}$ \\
\hline & \multicolumn{5}{|c|}{$\left(\mathrm{g} \mathrm{kg}^{-1}\right)$} & \multicolumn{5}{|c|}{$\left(\mathrm{mg} \mathrm{kg}^{-1}\right)$} \\
\hline MCC & 25.0 & 2.0 & 12.0 & 4.1 & 1.6 & 49.0 & 8.0 & 86.1 & 153.0 & 18.7 \\
\hline $\mathrm{OR} 00+\mathrm{AR} 00$ & $16.4^{* * *}$ & $0.9^{* * *}$ & $4.7^{* * *}$ & $4.4^{\mathrm{ns}}$ & $1.07^{* * *}$ & $32.3^{* * *}$ & $4.3^{\mathrm{ns}}$ & $275.7^{* * *}$ & $791.3^{* * *}$ & $39.0^{\text {ns }}$ \\
\hline OR00 + AR 15 & $17.5^{* * *}$ & $1.0^{* * *}$ & $6.0^{* * *}$ & $3.4^{\mathrm{ns}}$ & $1.47^{\mathrm{ns}}$ & $38.2^{\mathrm{ns}}$ & $14.0^{\text {ns }}$ & $307.5^{* * *}$ & $772.3^{* * *}$ & $81.7^{\mathrm{ns}}$ \\
\hline OR00 + AR30 & $18.0^{* * *}$ & $1.1^{* * *}$ & $5.3^{*+* *}$ & $3.6^{\mathrm{ns}}$ & $1.33^{\mathrm{ns}}$ & $27.2^{* * *}$ & $11.3^{\mathrm{ns}}$ & $186.0^{\mathrm{ns}}$ & $674.0^{* * *}$ & $45.0^{\text {ns }}$ \\
\hline OR00 + AR45 & $18.0^{* * *}$ & $1.0^{* * *}$ & $6.3^{* * *}$ & $3.1^{\mathrm{ns}}$ & $0.93^{* * *}$ & $32.5^{* * *}$ & $10.0^{\mathrm{ns}}$ & $166.3^{\text {ns }}$ & $591.3^{\mathrm{ns}}$ & $72.0^{\text {ns }}$ \\
\hline OR $16+$ AR00 & $20.3^{\text {ns }}$ & $0.9^{* * *}$ & $4.7^{* * *}$ & $3.5^{\mathrm{ns}}$ & $1.47^{\mathrm{ns}}$ & $37.7^{\mathrm{ns}}$ & $10.0^{\text {ns }}$ & $192.3^{\text {ns }}$ & $650.7^{* * *}$ & $29.0^{\text {ns }}$ \\
\hline OR16 + AR15 & $19.6^{\mathrm{ns}}$ & $0.9^{* * *}$ & $6.7^{* * *}$ & $3.1^{\mathrm{ns}}$ & $1.07^{* * *}$ & $28.0^{* * *}$ & $16.3^{\text {ns }}$ & $144.7^{\mathrm{ns}}$ & $610.7^{\mathrm{ns}}$ & $122.7^{* * *}$ \\
\hline OR16 + AR30 & $18.2^{* * *}$ & $0.9^{* * *}$ & $4.0^{*+* *}$ & $2.9^{\mathrm{ns}}$ & $1.27^{\mathrm{ns}}$ & $45.9^{\text {ns }}$ & $11.5^{\mathrm{ns}}$ & $640.4^{* * *}$ & $864.5^{* * *}$ & $104.5^{\mathrm{ns}}$ \\
\hline OR16 + AR45 & $19.6^{\mathrm{ns}}$ & $1.1^{* * *}$ & $6.7^{* * *}$ & $2.5^{\mathrm{ns}}$ & $0.97^{* * *}$ & $25.3^{* * *}$ & $7.0^{\text {ns }}$ & $108.0^{\mathrm{ns}}$ & $414.0^{\mathrm{ns}}$ & $50.7^{\mathrm{ns}}$ \\
\hline OR 32 + AR00 & $15.4^{* * *}$ & $0.8^{* * *}$ & $4.0^{* * *}$ & $2.5^{\mathrm{ns}}$ & $1.17^{\mathrm{ns}}$ & $34.1^{\mathrm{ns}}$ & $9.7^{\mathrm{ns}}$ & $116.3^{\mathrm{ns}}$ & $619.0^{\mathrm{ns}}$ & $39.0^{\text {ns }}$ \\
\hline OR32 + AR15 & $14.8^{* * *}$ & $0.6^{* * *}$ & $4.7^{* * *}$ & $2.5^{\mathrm{ns}}$ & $1.0^{* * *}$ & $49.2^{\mathrm{ns}}$ & $8.0^{\mathrm{ns}}$ & $145.6^{\mathrm{ns}}$ & $572.0^{\mathrm{ns}}$ & $62.3^{\mathrm{ns}}$ \\
\hline OR32 + AR30 & $17.5^{* * *}$ & $1.1^{* * *}$ & $6.7^{* * *}$ & $3.9^{\text {ns }}$ & $1.03^{* * *}$ & $23.6^{* * *}$ & $22.0^{\text {ns }}$ & $252.7^{\mathrm{ns}}$ & $748.0^{* * *}$ & $104.0^{\text {ns }}$ \\
\hline OR32 + AR45 & $18.2^{* * *}$ & $1.1^{* * *}$ & $7.3^{* * *}$ & $2.9^{\text {ns }}$ & $1.13^{\mathrm{ns}}$ & $12.7^{* * *}$ & $29.0^{\text {ns }}$ & $189.0^{\text {ns }}$ & $530.7^{\mathrm{ns}}$ & $103.0^{\text {ns }}$ \\
\hline $\mathrm{P}$ value & 0.0001 & 0.0001 & 0.8010 & 0.1162 & 0.0012 & 0.0002 & 0.4800 & 0.0038 & 0.0003 & 0.0217 \\
\hline Overall medial & 18.34 & 1.03 & 6.08 & 3.26 & 1.23 & 33.52 & 12.40 & 216.18 & 614.73 & 67.04 \\
\hline CV (\%) & 13 & 17 & 21 & 27 & 16 & 19 & 89 & 34 & 32 & 53 \\
\hline
\end{tabular}

ns: not significant values; ${ }^{* *}$ : significant difference between the means of the treatments and control (MCC) by Dunnett's test to $p<0.05$, respectively. OR: organic residue; AR: agroindustrial residue; MCC: seedlings collected in the field. 
Table 3. Mean values of foliar concentration of N, P, K, Ca, Mg, S, B, Cu, Fe, Mn, and Zn in Dipteryx alata seedlings for the residue treatments (experimental site). F values and coefficient of variation $(\mathrm{CV})$ are also shown.

\begin{tabular}{|c|c|c|c|c|c|c|c|c|c|c|}
\hline FV & $\mathbf{N}$ & $\mathbf{P}$ & $\mathbf{K}$ & $\mathrm{Ca}$ & $\mathrm{Mg}$ & B & $\mathbf{C u}^{*}$ & $\mathrm{Fe}$ & Mn & $\mathrm{Zn}$ \\
\hline & \multicolumn{5}{|c|}{$\left(\mathrm{g} \mathrm{kg}^{-1}\right)$} & \multicolumn{5}{|c|}{$\left(\mathrm{mg} \mathrm{kg}^{-1}\right)$} \\
\hline \multicolumn{11}{|c|}{ Organic residue (OR) } \\
\hline $0 \mathrm{t} \mathrm{ha}^{-1}$ & $17.4 \mathrm{ab}$ & 1.0 & 5.6 & 3.6 & 1.2 & 32.6 & 9.9 & 233.9 & 707.2 & 59.4 \\
\hline $16 \mathrm{t} \mathrm{ha}^{-1}$ & $19.4 \mathrm{a}$ & 0.9 & 5.5 & 3.0 & 1.2 & 34.2 & 11.2 & 271.3 & 634.9 & 76.7 \\
\hline $32 \mathrm{t} \mathrm{ha}^{-1}$ & $16.5 b$ & 0.9 & 5.7 & 3.0 & 1.1 & 29.9 & 17.1 & 175.9 & 617.4 & 77.1 \\
\hline \multicolumn{11}{|c|}{ Agroindustrial residue (AR) } \\
\hline $0 \mathrm{t} \mathrm{ha}^{-1}$ & 17.3 & 0.9 & 4.4 & 3.5 & 1.3 & 34.7 & 8.0 & 194.8 & 687.0 & 35.7 \\
\hline $15 \mathrm{t} \mathrm{ha}^{-1}$ & 17.3 & 0.8 & 5.8 & 3.0 & 1.2 & 38.5 & 12.8 & 199.2 & 651.7 & 88.9 \\
\hline $30 \mathrm{t} \mathrm{ha}^{-1}$ & 17.8 & 1.0 & 5.3 & 3.4 & 1.2 & 32.3 & 14.9 & 359.6 & 762.2 & 84.5 \\
\hline $45 \mathrm{t} \mathrm{ha}^{-1}$ & 18.6 & 1.1 & 6.8 & 2.8 & 1.0 & 23.5 & 15.3 & 154.4 & 512.0 & 75.2 \\
\hline \multicolumn{11}{|c|}{ F value } \\
\hline $\mathrm{BL}^{\# \#}$ & $0.89^{\text {ns }}$ & $0.54^{\mathrm{ns}}$ & $3.90^{\text {ns }}$ & $2.35^{\mathrm{ns}}$ & $0.29^{\text {ns }}$ & $4.07^{\mathrm{ns}}$ & $1.73^{\text {ns }}$ & $1.97^{\mathrm{ns}}$ & $1.16^{\mathrm{ns}}$ & $2.22^{\mathrm{ns}}$ \\
\hline OR & $2.57^{\mathrm{ns}}$ & $0.42^{\mathrm{ns}}$ & $0.40^{\mathrm{ns}}$ & $3.33^{\mathrm{ns}}$ & $1.05^{\mathrm{ns}}$ & $3.39^{\mathrm{ns}}$ & $0.59^{\text {ns }}$ & $10.71^{* *}$ & $0.28^{\mathrm{ns}}$ & $0.73^{\text {ns }}$ \\
\hline AR & $0.87^{\text {ns }}$ & $2.64^{\mathrm{ns}}$ & $5.95^{*}$ & $0.92^{\mathrm{ns}}$ & $1.33^{\text {ns }}$ & $8.31^{* *}$ & $1.52^{\mathrm{ns}}$ & $25.32^{* *}$ & $3.06^{\mathrm{ns}}$ & $2.09^{\text {ns }}$ \\
\hline ORxAR & $0.99^{\text {ns }}$ & $1.61^{\mathrm{ns}}$ & $1.29^{\mathrm{ns}}$ & $0.99^{\text {ns }}$ & $6.05^{* *}$ & $7.12^{* *}$ & $1.36^{\mathrm{ns}}$ & $8.208^{* *}$ & $1.11^{\mathrm{ns}}$ & $3.73^{*}$ \\
\hline $\mathrm{CV}(\%)$ & 12 & 16 & 28 & 30 & 12 & 22 & 36 & 40 & 24 & 36 \\
\hline
\end{tabular}

\#Transformed data to $\sqrt{ }(x+0.5)$. Means followed by the same letter in columns by source of variation do not differ significantly at $5 \%$ probability by the Tukey's test. ns: not significant values; ${ }^{*}$ and ": values significant at $p<0.05$ and $p<0.01$, respectively. ${ }^{* *}$ : the results for blocks were not significant.

The application of AR and OR did not lead to any significant increase in the $P$ concentration of leaves, possibly due to $P$ fixation in the soil reducing its availability to plants. The $\mathrm{K}$ concentration of leaves ranged from 4.4 to $6.8 \mathrm{~g} \mathrm{~kg}^{-1}$ between the lowest and the highest AR dose, respectively. Thus, $\mathrm{K}$ concentration showed a strong response to AR doses, increasing linearly $\left(\hat{y}^{* *}=3,944+0,655 x ; R^{2}=0.7609\right)$. For every $15 \mathrm{Mg} \mathrm{ha}^{-1} \mathrm{AR}$ added, there were foliar K concentration increments of $9.825 \mathrm{mg} \mathrm{kg}^{-1}$ biomass (Table 3). The foliar concentration of $\mathrm{Ca}, \mathrm{Mg}$, and $\mathrm{Cu}$ in introduced plants was similar to that in CER seedlings. However, the applied residue had no effect on $\mathrm{Ca}$ and $\mathrm{Cu}$ concentrations (Table 3). In comparison, foliar $\mathrm{Mg}$ was influenced by the quantity of applied residue, but showed no clear pattern (Table 4).

After 2 years of adding residues to the soil at the experimental area, soil $\mathrm{P}, \mathrm{MO}, \mathrm{K}, \mathrm{Ca}, \mathrm{Mg}, \mathrm{Cu}, \mathrm{Fe}, \mathrm{Mn}$, and $\mathrm{Zn}$ contents, as well as $\mathrm{pH}$, were low in the experimental area
(Table 5). Except for P, $\mathrm{K}$, and $\mathrm{pH}$, all other soil elements were lower in the experimental area compared to CER (Table 5). $\mathrm{K}^{+}$ranged from 0.4 to $1.5 \mathrm{mmol} \mathrm{dm}^{-3}$ in the soil treated with residues (Table 5 ). While these values were very low compared to other studies ( 3 to $6.0 \mathrm{mmol} \mathrm{dm}^{-3}$ ) (Raij et al., 2001), the recorded values were sufficient to explain the increase in foliar $\mathrm{K}$ concentration. The nutrient concentration of seedlings presented no correlation with the levels found in the soil (Viani et al., 2014). Soil samples were collected at $0.20 \mathrm{~m}$ depth, with seedlings growing in the soil for 12 months; thus, results could be compromised, because roots (even within 12 months) expanded to depths greater than $0.20 \mathrm{~m}$. Therefore, a soil depth greater than $0.20 \mathrm{~m}$ could be analyzed to evaluate the soil-plant relationship more closely. Furthermore, the extraction method used (ion exchange resin) could not adequately represent the potential of $D$. alata to use soil nutrients.

Table 4. Influence of organic (OR) and agroindustrial residues (AR) added to the soil on the foliar concentration of Mg, B, and Fe in Dipteryx alata seedlings.

\begin{tabular}{|c|c|c|c|c|c|c|c|}
\hline \multicolumn{8}{|c|}{$\operatorname{AR}\left(\mathrm{Mg} \mathrm{ha}^{-1}\right)$} \\
\hline OR & 00 & 15 & 30 & 45 & $\mathbf{F}$ & \multirow{2}{*}{ Regression equations } & \multirow{2}{*}{$\mathbf{R}^{2}$} \\
\hline \multicolumn{6}{|c|}{$\mathrm{Mg}\left(\mathrm{g} \mathrm{kg}^{-1}\right)$} & & \\
\hline 00 & 1.1 & $1.5 \mathrm{a}$ & 1.4 & 1.0 & $4.25^{*}$ & $\begin{array}{c}\hat{y}=0.367+0.947 x-0.200 x^{2} \\
t=1.115^{\text {ns }} 3.155^{* *}-3.386^{* *}\end{array}$ & 0.9800 \\
\hline 16 & 1.2 & $1.1 \mathrm{ab}$ & 1.3 & 1.0 & $3.67^{*}$ & $\begin{array}{c}\hat{y}=1.56-0.13 x \\
t=10.759^{* *}-2.536^{*}\end{array}$ & 0.8421 \\
\hline 32 & 1.5 & $1.0 \mathrm{~b}$ & 1.0 & 1.1 & $0.42^{\text {ns }}$ & $\hat{\mathrm{y}}^{\mathrm{ns}}$ & \\
\hline $\mathrm{F}$ & $3.210^{\mathrm{ns}}$ & $4.651^{\star}$ & $2.043^{\mathrm{ns}}$ & $0.753^{\mathrm{ns}}$ & & & \\
\hline \multicolumn{8}{|c|}{ B $\left(\mathrm{mg} \mathrm{kg}^{-1}\right)$} \\
\hline 00 & 32.3 & $38.2 \mathrm{ab}$ & $27.2 \mathrm{~b}$ & $32.4 \mathrm{a}$ & $1.42^{\mathrm{ns}}$ & $\hat{y}^{\mathrm{ns}}$ & \\
\hline
\end{tabular}


Table 4. Continued...

\begin{tabular}{|c|c|c|c|c|c|c|c|}
\hline \multicolumn{8}{|c|}{$\operatorname{AR}\left(\mathrm{Mg} \mathrm{ha}^{-1}\right)$} \\
\hline OR & 00 & 15 & 30 & 45 & $\mathbf{F}$ & \multirow{2}{*}{ Regression equations } & \multirow{2}{*}{$\mathbf{R}^{2}$} \\
\hline \multicolumn{6}{|c|}{$\operatorname{Mg}\left(\mathrm{g} \mathrm{kg}^{-1}\right)$} & & \\
\hline 16 & 34.1 & $28.0 \mathrm{~b}$ & $45.9 \mathrm{a}$ & $25.3 \mathrm{ab}$ & $6.25^{* *}$ & $\hat{y}^{\mathrm{ns}}$ & \\
\hline 32 & 37.7 & $49.2 \mathrm{a}$ & $23.6 \mathrm{~b}$ & $12.7 \mathrm{~b}$ & $16.99^{* *}$ & $\begin{array}{l}\hat{y}=52.33-8.97 x \\
t=11.316^{* *}-5.314^{* *}\end{array}$ & 0.5541 \\
\hline $\mathrm{F}$ & $0.519^{\mathrm{ns}}$ & $7.903^{* *}$ & $10.056^{* *}$ & $7.058^{* *}$ & & & \\
\hline \multicolumn{8}{|c|}{$\mathrm{Fe}\left(\mathrm{mg} \mathrm{kg}^{-1}\right)$} \\
\hline 00 & 275.7 & $307.5 \mathrm{a}$ & $186.0 \mathrm{~b}$ & 166.3 & $2.43^{\mathrm{ns}}$ & $\hat{y}^{\mathrm{ns}}$ & \\
\hline 16 & 192.3 & $144.6 \mathrm{ab}$ & $640.7 \mathrm{a}$ & 108.0 & $32.05^{* *}$ & $\begin{array}{c}\hat{Y}=-21.51+148.63 \mathrm{x}-23.22 \mathrm{x}^{2} \\
\mathrm{t}=-0.176^{\text {ns }} 1.334^{\text {ns }}-1.059^{\text {ns }}\end{array}$ & 0.7065 \\
\hline 32 & 116.3 & $145.6 \mathrm{ab}$ & $252.7 \mathrm{~b}$ & 189.0 & $1.82^{\mathrm{ns}}$ & $\hat{y}^{\mathrm{ns}}$ & \\
\hline $\mathrm{F}$ & $3.30^{\text {ns }}$ & $4.57^{*}$ & $31.26^{* *}$ & $0.91^{\mathrm{ns}}$ & & & \\
\hline \multicolumn{8}{|c|}{$\mathrm{Zn}\left(\mathrm{mg} \mathrm{kg}^{-1}\right)$} \\
\hline 00 & 39.0 & 81.7 & 45.0 & 72.0 & $1.96^{\mathrm{ns}}$ & $\hat{y}^{\text {ns }}$ & \\
\hline 16 & 29.0 & 122.7 & 104.5 & 50.7 & $8.93^{* *}$ & $\begin{array}{c}\hat{y}=-119.37+189.06 x-36.87 x^{2} \\
t=-2.905^{\text {ns }} 5.044^{* *}-4.997^{* *}\end{array}$ & 0.9503 \\
\hline 32 & 39.0 & 62.3 & 104.0 & 103.0 & $4.69^{*}$ & $\begin{aligned} \hat{y} & =18.67+23.37 x \\
t & =1.033^{\text {ns }} 3.540^{* *}\end{aligned}$ & 0.8910 \\
\hline $\mathrm{F}$ & $0.043^{\mathrm{ns}}$ & $1.229^{\mathrm{ns}}$ & $1.516^{\mathrm{ns}}$ & $0.897^{\mathrm{ns}}$ & & & \\
\hline
\end{tabular}

Means followed by the same letter in columns by source of variation do not differ significantly at $5 \%$ probability by the Tukey's test. ${ }^{\text {ns: }}$ not significant values; ${ }^{*}$ and ${ }^{* *}$ : values significant at $p<0.05$ and $p<0.01$, respectively.

Despite of the lack of a direct relationship between element concentrations in leaves and soil, the height and diameter of plants in the experimental area were positively influenced by the use of residues (Table 5). Plant height ranged from 46.0 to $62.9 \mathrm{~cm}$ in OR treatments and between
41.3 and $72.3 \mathrm{~cm}$ in AR treatments, with a linear increase $\left(\hat{y}^{* *}=30.092+10.562 x ; R^{2}=0.9947\right)$ (Table 6). The stem diameter ranged between 12.7 and $15.7 \mathrm{~mm}$ in the $\mathrm{OR}$ treatment and from 12.3 to $16.6 \mathrm{~mm}$ in the AR treatment, with a linear increase $\left(\hat{y}^{* *}=11.109+1.358 x, R^{2}=0.9787\right)$.

Table 5. Mean values of $\mathrm{P}, \mathrm{MO}, \mathrm{pH}, \mathrm{K}, \mathrm{Ca}, \mathrm{Mg}, \mathrm{H}+\mathrm{Al}, \mathrm{Al}, \mathrm{B}, \mathrm{Cu}, \mathrm{Fe}, \mathrm{Mn}$, and $\mathrm{Zn}$ in the soil for residue treatments (experimental site) and natural site (remnants of Cerrado). F values and coefficients of variation (CV) are presented.

\begin{tabular}{|c|c|c|c|c|c|c|c|c|c|c|c|c|c|}
\hline \multirow{2}{*}{ Treatments } & $\mathbf{P}$ & MO & pH & $\mathbf{K}$ & $\mathrm{Ca}$ & $\mathrm{Mg}$ & $\mathbf{H}+\mathbf{A} \mathbf{l}$ & $\mathbf{A l}^{*}$ & B & $\mathrm{Cu}^{*}$ & $\mathrm{Fe}^{*}$ & $\mathbf{M n}^{*}$ & $\mathbf{Z n}^{*}$ \\
\hline & $\left(\mathrm{mg} \mathrm{dm}^{-3}\right)$ & $\left(\mathrm{g} \mathrm{dm}^{-3}\right)$ & $\left(\mathrm{CaCl}_{2}\right)$ & \multicolumn{5}{|c|}{$\left(\mathrm{mmol}_{\mathrm{c}} \mathrm{dm}^{-3}\right)$} & \multicolumn{5}{|c|}{$\left(\mathrm{mg} \mathrm{dm}^{-3}\right)$} \\
\hline SCC & 2.3 & 23.3 & 4.6 & 1.2 & 12.3 & 9.7 & 36.7 & 4.3 & 0.3 & 3.1 & 59.3 & 43.5 & $0.7^{* * *}$ \\
\hline OR00 + AR00 & 1.0 & $8.3^{* * *}$ & $4.3^{\mathrm{ns}}$ & $0.4^{* * *}$ & $1.3^{* * *}$ & $1.3^{* * *}$ & $28.0^{* * *}$ & $9.3^{* * *}$ & $0.1^{* * *}$ & $0.6^{* *}$ & $2.0^{* * *}$ & $12.5^{* * *}$ & $0.1^{* * *}$ \\
\hline OR00 + AR15 & 1.0 & $9.0^{* * *}$ & $4.4^{\mathrm{ns}}$ & $0.6^{\mathrm{ns}}$ & $3.7^{* * *}$ & $2.6^{* * *}$ & $25.0^{* * *}$ & $4.7^{\mathrm{ns}}$ & $0.1^{* * *}$ & $0.6^{* * *}$ & $2.7^{* * *}$ & $11.8^{* * *}$ & $0.1^{* * *}$ \\
\hline $\mathrm{OR} 00+\mathrm{AR} 30$ & 1.0 & $8.3^{* * *}$ & $4.4^{\mathrm{ns}}$ & $0.5^{* *+}$ & $4.6^{* * *}$ & $1.7^{* * *}$ & $25.3^{* * *}$ & $4.7^{\mathrm{ns}}$ & $0.1^{* * *}$ & $0.6^{4+*}$ & $2.0^{* * *}$ & $11.1^{*+*+}$ & $0.1^{*+*}$ \\
\hline OR00 + AR45 & 1.0 & $8.7^{* * *}$ & $4.8^{\mathrm{ns}}$ & $1.1^{\mathrm{ns}}$ & $8.7^{\mathrm{ns}}$ & $3.7^{* * *}$ & $20.3^{* * *}$ & $2.0^{\mathrm{ns}}$ & $0.1^{* * *}$ & $0.6^{* * *}$ & $2.3^{* * *}$ & $10.1^{* * *}$ & $0.1^{* * *}$ \\
\hline OR16 + AR00 & 1.0 & $9.7^{* * *}$ & $4.3^{\mathrm{ns}}$ & $0.6^{\mathrm{ns}}$ & $2.3^{* * *}$ & $1.6^{* * *}$ & $28.0^{* * *}$ & $9.3^{* * *}$ & $0.1^{* * *}$ & $0.8^{* * *}$ & $3.0^{* * *}$ & $14.1^{* * *}$ & $0.2^{* * *}$ \\
\hline OR16 + AR15 & 1.0 & $9.7^{* * *}$ & $4.4^{\mathrm{ns}}$ & $0.6^{\mathrm{ns}}$ & $4.3^{* * *}$ & $4.0^{* * *}$ & $26.3^{* * *}$ & $6.3^{\text {ns }}$ & $0.1^{* * *}$ & $0.7^{* * *}$ & $2.3^{* * *}$ & $11.4^{* * *}$ & $0.2^{* * *}$ \\
\hline OR16 + AR30 & 1.0 & $9.7^{* * *}$ & $4.6^{\mathrm{ns}}$ & $1.0^{\mathrm{ns}}$ & $5.7^{* * *}$ & $3.0^{* * *}$ & $23.0^{* * *}$ & $4.3^{\mathrm{ns}}$ & $0.1^{* * *}$ & $0.7^{* * *}$ & $3.3^{+* x+}$ & $11.8^{* * *}$ & $0.1^{* * *}$ \\
\hline OR16 + AR45 & 1.0 & $9.3^{* * *}$ & $4.7^{\mathrm{ns}}$ & $1.2^{\mathrm{ns}}$ & $8.3^{\mathrm{ns}}$ & $3.3^{* * *}$ & $21.7^{* * *}$ & $2.7^{\mathrm{ns}}$ & $0.1^{* * *}$ & $0.6^{* * *}$ & $2.7^{* * *}$ & $10.8^{* * *}$ & $0.1^{* * *}$ \\
\hline OR $32+$ AR00 & 1.0 & $9.0^{* * *}$ & $4.4^{\mathrm{ns}}$ & $0.8^{\mathrm{ns}}$ & $4.3^{* * *}$ & $2.3^{* * *}$ & $26.0^{* * *}$ & $5.3^{\mathrm{ns}}$ & $0.1^{* * *}$ & $0.6^{*+*}$ & $2.3^{* * *}$ & $10.3^{* * *}$ & $0.2^{* * *}$ \\
\hline OR32 + AR15 & 1.0 & $9.0^{* * *}$ & $4.5^{\mathrm{ns}}$ & $0.9^{\text {ns }}$ & $3.7^{* * *}$ & $2.6^{* * *}$ & $25.0^{* * *}$ & $4.7^{\mathrm{ns}}$ & $0.1^{* * *}$ & $0.7^{* * *}$ & $2.7^{* * *}$ & $13.1^{* * *}$ & $0.3^{* * *}$ \\
\hline OR32 + AR30 & 1.0 & $9.3^{* * *}$ & $4.8^{\mathrm{ns}}$ & $1.5^{\mathrm{ns}}$ & $8.7^{\mathrm{ns}}$ & $4.3^{* * *}$ & $21.0^{* * *}$ & $1.7^{\mathrm{ns}}$ & $0.1^{* * *}$ & $0.7^{* * *}$ & $3.7^{*+* x}$ & $9.9^{* * *}$ & $0.1^{* * *}$ \\
\hline $\mathrm{OR} 32+\mathrm{AR} 45$ & 1.0 & $8.3^{* * *}$ & $4.6^{\mathrm{ns}}$ & $1.3^{\mathrm{ns}}$ & $7.3^{* * *}$ & $3.7^{* * *}$ & $21.7^{* * *}$ & $3.3^{\mathrm{ns}}$ & $0.1^{* * *}$ & $0.6^{* * *}$ & $2.0^{* * *}$ & $10.9^{* * *}$ & $0.3^{* * *}$ \\
\hline
\end{tabular}


Table 5. Continued...

\begin{tabular}{|c|c|c|c|c|c|c|c|c|c|c|c|c|c|}
\hline & $\mathbf{P}$ & MO & pH & $\mathbf{K}$ & $\mathrm{Ca}$ & $\mathrm{Mg}$ & $\mathbf{H}+\mathbf{A l}$ & $\mathbf{A l}^{*}$ & B & $\mathbf{C u}^{*}$ & $\mathrm{Fe}^{*}$ & $\mathbf{M n}^{*}$ & $\mathbf{Z n}^{*}$ \\
\hline 10. & $\left(\mathrm{mg} \mathrm{dm}^{-3}\right)$ & $\left(\mathrm{g} \mathrm{dm}^{-3}\right)$ & $\left(\mathrm{CaCl}_{2}\right)$ & \multicolumn{5}{|c|}{$\left(\mathrm{mmol}_{\mathrm{c}} \mathrm{dm}^{-3}\right)$} & \multicolumn{5}{|c|}{$\left(\mathrm{mg} \mathrm{dm}^{-3}\right)$} \\
\hline$P$ value & 0.2841 & 0.0001 & 0.7131 & 0.0970 & 0.0001 & 0.0001 & 0.0001 & 0.5670 & 0.0001 & 0.0001 & 0.0001 & 0.0001 & 0.0001 \\
\hline $\begin{array}{l}\text { Overall } \\
\text { average }\end{array}$ & 1.11 & 10.13 & 4.53 & 0.89 & 5.79 & 3.38 & 25.23 & 4.82 & 0.13 & 0.83 & 6.94 & 14.01 & 0.20 \\
\hline $\mathrm{CV} \%$ & 14 & 9 & 3 & 32 & 29 & 42 & 8 & 31 & 12 & 29 & 35 & 24 & 65 \\
\hline
\end{tabular}

\#Transformed data to $\sqrt{ }(\mathrm{x}+0.5)$. ns: not significant values; ${ }^{* *}$ : significant difference between the means of the treatments and mean of control (MCC) using Dunnett's test to $p<0.05$, respectively. OR: organic residue; AR: agroindustrial residue, SCC: soil collected from the Cerrado.

Table 6. Mean values for plant height and stem diameter in the residue treatments.

\begin{tabular}{|c|c|c|}
\hline \multirow[t]{2}{*}{ Treatments } & Plant height & $\begin{array}{c}\text { Stem } \\
\text { diameter }\end{array}$ \\
\hline & $(\mathrm{cm})$ & $(\mathrm{mm})$ \\
\hline \multicolumn{3}{|c|}{ Organic residue (OR) } \\
\hline $0 \mathrm{Mg} \mathrm{ha}^{-1}$ & $46.0 \mathrm{~b}$ & $12.7 \mathrm{~b}$ \\
\hline $16 \mathrm{Mg} \mathrm{ha}^{-1}$ & $62.3 \mathrm{a}$ & $15.4 \mathrm{a}$ \\
\hline $32 \mathrm{Mg} \mathrm{ha}^{-1}$ & $62.9 \mathrm{a}$ & $15.7 \mathrm{a}$ \\
\hline \multicolumn{3}{|c|}{ Agroindustrial residue (AR) } \\
\hline $0 \mathrm{Mg} \mathrm{ha}^{-1}$ & 41.3 & 12.3 \\
\hline $15 \mathrm{Mg} \mathrm{ha}^{-1}$ & 49.8 & 14.1 \\
\hline $30 \mathrm{Mg} \mathrm{ha}^{-1}$ & 62.5 & 14.9 \\
\hline $45 \mathrm{Mg} \mathrm{ha}^{-1}$ & 72.3 & 16.6 \\
\hline \multicolumn{3}{|c|}{ F value } \\
\hline $\mathrm{BL}$ & $0.54^{\mathrm{ns}}$ & $1.96^{\mathrm{ns}}$ \\
\hline OR & $7.29^{*}$ & $12.70^{*}$ \\
\hline AR & $24.88^{* *}$ & $5.82^{*}$ \\
\hline $\mathrm{OR} \times \mathrm{AR}$ & $1.346^{\mathrm{ns}}$ & $1.28^{\mathrm{ns}}$ \\
\hline $\mathrm{CV}(\%)$ & 52 & 37 \\
\hline
\end{tabular}

Means followed by the same letter in columns by source of variation do not differ significantly at $5 \%$ probability by the Tukey's test. ns: non-significant values; ${ }^{*}$ and $^{* *}$ : values significant at $p<0.05$ and $p<0.01$, respectively.

\section{DISCUSSION}

Viani et al. (2014) documented $\mathrm{N}$ concentrations ranging between 16 and $29 \mathrm{~g} \mathrm{~kg}^{-1}$ for several plant species from the Brazilian Savannah, including Mabea fistulifera and Copaifera langsdorfii. Drechsel \& Zech (1991) found that the intermediate $\mathrm{N}$ concentration in the leaves of legumes, such as Acacia auriculiformis, ranges between 16 and $28 \mathrm{~g} \mathrm{~kg}^{-1}$ under a variety of treatments. In the current study, P concentration of leaves from CER plants was low, but it was, on average, $70 \%$ higher than that of $\mathrm{P}$ concentration of leaves exposed to the residue treatments (Table 2). This result demonstrates that $D$. alata has low $\mathrm{P}$ demand; however, there was insufficient $\mathrm{P}$ in the soil of both CER and experimental area, with residues failing to enhance P levels in the soil and plants (Tables 3 and 4). In particular, most soils of the Brazilian Savannah are acidic with low nutrient content, especially $\mathrm{P}$, which might restrict the plant development (Venterink, 2011). The acidity of the soil at the experimental site has not been remedied, with $\mathrm{pH}$ values of 4.3 to 4.8 . Consequently, part of the $\mathrm{P}$ from the residues might have been fixed by soil oxides (Gérard, 2016).

Yang et al. (2012) showed that conventional chemical nutrient extraction from soil was significantly related to the amount of $\mathrm{P}$ absorbed by plants. The data from the current study corroborated this result, with seedlings presenting $P$ values similar to those found in the soil (Table 5). This phenomenon is logical because $\mathrm{P}$ is an element that has low mobility in the soil (Shen et al., 2011). Ferreira et al. (2012) showed that the incorporation of ash in soil enhances fertility and growing conditions, reducing $\mathrm{Al}$ and increasing $\mathrm{pH}$. Therefore, the availability of other elements (including $\mathrm{Mg}, \mathrm{P}$, and $\mathrm{K}$ ) to plants also increase.

Foliar Mg levels were variable (Table 3). In the presence of $15 \mathrm{Mg} \mathrm{ha}^{-1} \mathrm{AR}$, foliar $\mathrm{Mg}$ content ranged between 1.5 and $1.0 \mathrm{~g} \mathrm{~kg}^{-1}$, representing the lowest and highest dose of OR, respectively. Similar variation was detected in the presence and absence of $16 \mathrm{Mg} \mathrm{ha}^{-1} \mathrm{OR}$. Viani et al. (2014) found that the foliar Mg of several Brazilian Savannah species ranged between 2 and $8 \mathrm{~g} \mathrm{~kg}^{-1}$. These values were similar to those observed for $D$. alata under most treatments in this study. However, in some parts, concentrations were still below the required by plants.

$\mathrm{Mg}$ leaf concentration is strongly correlated to soil $\mathrm{P}$ and $\mathrm{pH}$. Low $\mathrm{P}$ content in the soil inhibits $\mathrm{Mg}$ absorption, leading to low foliar Mg (Santos et al., 2006). Furthermore, an increase in the uptake and presence of $\mathrm{K}$ induces lower $\mathrm{Mg}$ absorption, which might explain the low levels of documented Mg.

Micronutrients showed great variability in this study, based on the coefficient of variation. Similarly, high variability was detected when comparing treatmentseedlings with those in CER (Tables 2 and 3), hampering the interpretation.

The application of $32 \mathrm{Mg} \mathrm{ha}^{-1} \mathrm{OR}$ combined with different doses of AR ( 0 to $45 \mathrm{Mg} \mathrm{ha}^{-1}$ ) caused B concentrations to decline in the plant, with $\mathrm{B}$ concentrations significantly decreasing with increasing AR dose (Table 4). 
Higher doses of both OR and AR generated foliar B concentrations of $12.7 \mathrm{mg} \mathrm{kg}^{-1}$ (Table 4), which was below the minimum of $20 \mathrm{mg} \mathrm{kg}^{-1}$ documented for some legumes (Drechsel \& Zech, 1991). Epstein \& Bloom (2005) state that B is highly variable in the tissues of some plants $\left(5-300 \mathrm{mg} \mathrm{kg}^{-1}\right)$, with no symptoms of toxicity.

There was high variability in the foliar concentration of Fe in relation to the applied OR and AR doses (Table 3), hampering the interpretation.

The Fe content of the soil did not show a direct relationship to foliar $\mathrm{Fe}$ in the experimental area; however, CER plants were strongly related (Tables 2 and 5). Thus, D. alata might reduce $\mathrm{Fe}^{+3}$ ions in the soil, increasing the absorption of $\mathrm{Fe}^{+3}$ and other nutrients.

Schmidt et al. (2014) showed the availability of Fe in the rhizosphere is limited by the low solubility and dissolution rate of inorganic iron compounds required for plant and microbial growth in the soil. Thus, Fe might have been solubilized from the agroindustrial residue in this study $\left(1540 \mathrm{mg} \mathrm{kg}^{-1}\right)$.

Seedlings introduced in the experimental area had Fe leaf contents above $100 \mathrm{mg} \mathrm{kg}^{-1}$, which is considered as intermediate for legumes (Drechsel \& Zech, 1991). The seedlings showed no symptoms of toxicity. Therefore, $D$. alata might be adapted to $\mathrm{Fe}$, leading to the production of nuts with high $\mathrm{Fe}$ content, with possible use in human food (Siqueira et al., 2012).

Statistically significant differences in the foliar concentration of Mn were observed in plants from the residue treatments and CER. In both cases, the concentration was very high (Table 2), being 414-864 $\mathrm{mg} \mathrm{kg}^{-1}$ in plants from the experimental plot and $138.4 \mathrm{mg} \mathrm{kg}^{-1}$ in plants from CER, suggesting D. alata is a hyperaccumulator species of $\mathrm{Mn}$. This behavior was also documented by Lambers et al. (2015), with high foliar Mn being detected in Proteaceae. In this study, the observed values fell within the range considered critical $\left(0.2\right.$ to $\left.3.5 \mathrm{mg} \mathrm{kg}^{-1}\right)$ by Krämer (2010). However, the plants in the current study presented no symptoms of toxicity, supporting their characterization as hyperaccumulators.

Lower foliar $\mathrm{Zn}$ concentrations were documented in the absence of AR, and in seedlings from the CER (Tables 2 and 3). In the experimental field, foliar $\mathrm{Zn}$ levels were higher in the presence of $\mathrm{AR}\left(\hat{\mathrm{y}}^{\star *}=-35.625+89.552 \mathrm{x}-15.625 \mathrm{x}^{2}\right.$; $\left.\mathrm{R}^{2}=0.9214\right)$.

This phenomenon might be attributed to the composition of ash (total $\mathrm{Zn} 12.4 \mathrm{mg} \mathrm{kg}^{-1}$ ) and it is associated with the identified mechanisms of solubilization. Unexpectedly, there was no relationship between $\mathrm{Zn}$ content in the soil and $\mathrm{Zn}$ concentration in the plant, which might be due to the extractor used to quantify $\mathrm{Zn}$ available in soil.

The observed decline in $\mathrm{Al}^{+3}$ (9.3 to $1.7 \mathrm{mmol} \mathrm{dm}^{-3}$ ) was desirable, because $\mathrm{Al}$ is toxic to plants (Haridasan, 1982).
Despite the added residues increasing soil $\mathrm{pH}$, it still remained acidic, which might interfere with the availability of other elements (Li \& Johnson, 2016), such as B, the availability of which is limited at $\mathrm{pH}<5$ (Lehto et al., 2010).

A dose of $16 \mathrm{Mg} \mathrm{ha}^{-1} \mathrm{OR}$ was sufficient to enhance the height and diameter of plants; thus, higher doses might not be necessary. Some studies showed that the absence of organic compounds affects the growth of hibiscus seedlings (Massa et al., 2016), with the observations made for the height and diameter of $D$. alata in this study supporting this finding (Table 6).

Due to the great variability in species characteristics and the heterogeneity of tropical regions, information on the nutritional requirements of Brazilian Savannah species and their ability to adapt to different environmental conditions is still limited, requiring focused studies in this area.

\section{CONCLUSIONS}

The D. alata is a Brazilian Savannah species that tolerates high leaf concentration of $\mathrm{Mn}$, and could be useful for the recovery of degraded areas, since it is not a nutritionally demanding plant.

The residues, ash and macrophytes, contributed to the revegetation of degraded area in the Brazilian Savannah.

\section{ACKNOWLEDGEMENTS}

We thank Glauber Stefan Barbosa for his assistance in producing the map.

This work was supported by Conselho Nacional de Desenvolvimento Científico e Tecnológico (CNPq) (grant number 561842/2010-8).

\section{SUBMISSION STATUS}

Received: 15 Mar. 2018

Accepted: 16 Apr. 2019

Associate editor: Rodrigo Studart Corrêa

(D) 0000-0002-9422-2629

\section{CORRESPONDENCE TO}

\section{Thaís Soto Boni}

Av. Brasil, 56, CEP 15385-000, Ilha Solteira, SP, Brasil e-mail: thais.sboni@gmail.com

\section{REFERENCES}

Agbenin JO, Abreu CA, Raij BV. Extraction of phytoavailable trace metals from tropical soils by mixed ion exchange resin modified with inorganic and organic ligands. Science of the Total Environment 1999; 227(2-3): 187-196. 10.1016/S0048-9697(99)00027-3 
Carnevale NJ, Montagnini F. Facilitating regeneration of secondary forests with the use of mixed and pure plantations of indigenous tree species. Forest Ecology and Management 2002; 163(1-3): 217-227. 10.1016/S0378-1127(01)00581-3

Drechsel P, Zech W. Foliar nutrient levels of broad-leaved tropical trees: a tabular review. Plant and Soil 1991; 131(1):29-46. 10.1007/ BF00010417

Epstein E, Bloom AJ. Mineral nutrition of higher plants: principles and perspectives. 2nd ed. Sunderland: Sinauer Associates; 2005.

Ferreira EPB, Fageriae NK, Didonet AD. Chemical properties of an Oxisol under organic management as influenced by application of sugarcane bagasse ash. Revista Ciência Agronômica 2012; 43(2): 228-236. 10.1590/S1806-66902012000200004

Gérard F. Clay minerals, iron/aluminum oxides, and their contribution to phosphate sorption in soils: a myth revisited. Geoderma 2016; 262: 213-226. 10.1016/j.geoderma.2015.08.036

Gunnarsson CC, Petersen CM. Water hyacinths as a resource in agriculture and energy production: a literature review. Waste Management 2007; 27(1): 117-129. 10.1016/j.wasman.2005.12.011

Haridasan M. Aluminum accumulation by some cerrado native species of central Brazil. Plant and Soil 1982; 65: 265-273. 10.1007/ BF02374657

Krämer U. Metal hyper accumulation in plants. Annual Review of Plant Biology 2010; 61: 517-534. 10.1146/annurev-arplant-042809-112156

Lambers H, Hayes PE, Laliberté E, Oliveira RS, Turner BL. Leaf manganese accumulation and phosphorus-acquisition efficiency. Trends in Plant Science 2015; 20(2): 83-90. 10.1016/j. tplants.2014.10.007

Le HD, Smith C, Herbohn J. What drives the success of reforestation projects in tropical developing countries? The case of the Philippines. Global Environmental Change 2014; 24: 334-348. 10.1016/j. gloenvcha.2013.09.010

Lehto T, Ruuhola T, Dell B. Boron in forest trees and forest ecosystems. Forest Ecology and Management 2010; 260(12): 2053-2069. 10.1016/j.foreco.2010.09.028

Lemos MRB, Siqueira EMA, Arruda SF, Zambiazi RC. The effect of roasting on the phenolic compounds and antioxidant potential of baru nuts (Dipteryx alata Vog.). Food Research International 2012; 48(2): 592-597. 10.1016/j.foodres.2012.05.027

Li W, Johnson CE. Relationships among $\mathrm{pH}$, aluminum solubility and aluminum complexation with organic matter in acid forest soils of the Northeastern United States. Geoderma 2016; 271: 234-242. 10.1016/j.geoderma.2016.02.030

Lorenzi H. Árvores brasileiras: manual de identificação e cultivo de plantas arbóreas do Brasil. Nova Odessa: Instituto Plantarum; 2000.

Massa D, Prisa D, Montoneri E, Battaglini D, Ginepro M, Negre M, Burchi G. Application of municipal biowaste derived products in Hibiscus cultivation: effect on leaf gaseous exchange activity, and plant biomass accumulation and quality. Scientia Horticulturae 2016; 205: 59-69.

Myers N, Mittermeier RA, Mittermeier CG, Fonseca GAB, Kent J. Biodiversity hotspots for conservation priorites. Nature 2000; 403: 853-858. 10.1038/35002501
Novak JM, Busscher WJ, Laird DL, Ahmedna M, Watts DW, Niandou MAS. Impact of biochar amendment on fertility of a southeastern coastal plain soil. Soil Science 2009; 174(2): 105-112.

Pedrol N, Puig CG, Souza P, Forján R, Vega FA, Asensio V et al. Soil fertility and spontaneous revegetation in lignite spoil banks under different amendments. Soil and Tillage Research 2010; 110(1): 134-142. 10.1016/j.still.2010.07.005

Raij BV, Andrade JC, Cantarella H, Quaggio JÁ, editors. Análise química para avaliação da fertilidade de solos tropicais. Campinas: IAC; 2001.

Raij BV, Quaggio JA, Silva NM. Extraction of phosphorus, potassium, calcium and magnesium by an ion-exchange resin procedure. Communications in Soil Science and Plant Analysis 1986; 17(5): 547-566. 10.1080/00103628609367733

Reid JL, Holl KD, Zahawi RA. Seed dispersal limitations shift over time in tropical forest restoration. Ecological Applications 2015; 25(4): 1072-1082. 10.1890/14-1399.1

Rodrigues GB, Maltoni KL, Cassiolato AMR. Dinâmica da regeneração do subsolo de áreas degradadas dentro do bioma cerrado. Revista Brasileira de Engenharia Agrícola e Ambiental 2007; 11(1): 73-80. 10.1590/S1415-43662007000100010

Sano SM, Ribeiro JF, Brito MA. Baru: biologia e uso. Planaltina: Embrapa Cerrado; 2004.

Santos UM Jr, Gonçalves JFC, Feldpausch TR. Growth, leaf nutrient concentration and photosynthetic nutrient use efficiency in tropical tree species planted in degraded areas in central Amazonia. Forest Ecology and Management 2006; 226(1-3): 299-309. 10.1016/j. foreco.2006.01.042

Schmidt HP, Kammann C, Niggli C, Evangelou MWH, Mackie KA, Abiven S. Biochar and biochar-compost as soil amendments to a vineyard soil: influences on plant growth, nutrient uptake, plant health and grape quality. Agriculture Ecosystems \& Environment 2014; 191: 117-123. 10.1016/j.agee.2014.04.001

Shen J, Yuan L, Zhang J, Li H, Bai Z, Chen X et al. Phosphorus dynamics: from soil to plant. Plant Physiology 2011; 156(3): 997-1005. 10.1104/pp.111.175232

Silva RR, Oliveira DR, Rocha GP, Vieira DL. Direct seeding of Brazilian savanna trees: effects of plant cover and fertilization on seedling establishment and growth. Restoration Ecology 2015; 23(4): 393-401. 10.1111/rec.12213

Siqueira EMA, Marin AMF, Cunha MSB, Fustinoni AM, Sant'Ana LP, Arruda SF. Consumption of baru seeds [Dipteryx alata Vog.], a Brazilian savanna nut, prevents iron-induced oxidative stress in rats. Food Research International 2012; 45(1): 427-433. 10.1016/j. foodres.2011.11.005

Sloan S, Jenkins CN, Joppa LN, Gaveau DLA, Laurance WF. Remaining natural vegetation in the global biodiversity hotspots. Biological Conservation 2014; 177: 12-24. 10.1016/j. biocon.2014.05.027

Statistical Analysis System - SAS. User's guide: statistics. Cary: SAS; 2012.

Tang G, Li K. Soil amelioration through afforestation and self-repair in a degraded valley-type savanna. Forest Ecology and Management 2014; 320: 13-20. 10.1016/j.foreco.2014.02.018 
Thomaz SM, Esteves SA, Murphy KJ, Santos AM, Caliman A, Guariento RD. Aquatic macrophytes in the tropics: ecology of populations and communities, impacts of invasions and use by man. In: Encyclopedia of Live Support Systems - EOLSS. Tropical biology and conservation management. Paris; 2008. Vol. 4. p. 27-60.

Universidade Estadual Paulista - Unesp. Canal clima da Unesp Ilha Solteira: área de hidráulica e irrigação [Internet]. Ilha Solteira: Unesp; [2015?] [cited 2015 July. Available from: https://bit.ly/2vU9OFT

Venterink HO. Does phosphorus limitation promote species-rich plant communities? Plant and Soil 2011; 345(1-2): 1-9. 10.1007/ s11104-011-0796-9
Viani RAG, Rodrigues RR, Dawson TE, Lambers H, Oliveira RS. Soil $\mathrm{pH}$ accounts for differences in species distribution and leaf nutrient concentrations of Brazilian woodland savannah and seasonally dry forest species. Perspectives in Plant Ecology, Evolution and Systematics 2014; 16(2): 64-74. 10.1016/j.ppees.2014.02.001

Yang XJ, Lai YL, Mo JY Shen H. A device for simulating soil nutrient extraction and plant uptake. Pedosphere 2012; 22(6): 755-763. 10.1016/S1002-0160(12)60061-7

Zheng FL. Effect of vegetation changes on soil erosion on the Loess Plateau. Pedosphere 2006; 16(4): 420-427. 10.1016/S10020160(06)60071-4 